\title{
The Possibility of Using BCI Applications in Physiotherapy
}

\author{
Zuzana Koudelkova ${ }^{1, *}$, Sarka Dankova ${ }^{2,3}$, Michal Filip" ${ }^{2,3}$, and Marcela Dabrovska ${ }^{2}$ \\ ${ }^{1}$ Tomas Bata University in Zlin, Faculty of Applied Informatics, Zlin, Czech Republic \\ ${ }^{2}$ University of Ostrava, Faculty of Medicine, Departament of Rehabilitation, Ostrava, Czech Republic \\ ${ }^{3}$ University Hospital of Ostrava, The Clinic of Rehabilitation and Physical, Ostrava, Czech Republic
}

\begin{abstract}
Brain-Computer Interface (BCI) is an interface connecting the human neural system and computer. This article explains the fundamental principles of BCI and devices, which can be controlled using electroencephalography (EEG). Firstly, this article describes Brain-Computer interface according to obtaining brain activity. After that, the applications of BCI are proposed, which can be used in clinical practice. In the experimental part, the external systems are defined. These external systems are operated by BCI technology. This technology is developed at the Department of Informatics and Artificial Intelligence of the Faculty of Applied Informatics, Tomas Bata University in Zlin. This BCI system contains EEG technology, which is responsible for scanning a brain activity with a fourteen-channel device developed by Emotiv company. In the near future, this design of peripheral systems can be involved in clinical practice in various medical branches, especially physiotherapy.
\end{abstract}

\section{Introduction}

Brain-Computer Interface is a system that enables detection and analysis of electric signals generated in the human brain. These brain signals translate into discrete computer form, which allows communication between electrical devices and the brain in real time. The transform and process these signals would partially recover communication of the impaired human organism with the surroundings. According to foreign publications, future potential could have a significant impact on clinical practice. This can be especially beneficial for rehabilitation treatment, patients after a stroke, a spinal cord injury, degenerative diseases of the nervous system, such as ALS, muscular dystrophy, etc. [1-3]

A neuron is the basic unit of the nervous system. The main function of this neuron is the transmission of the nerve impulse. The impulse is an electric signal generated by ion changes on the surface of the cell membrane. The groups of neurons create a network in the brain tissue to transform information for subsequent scanning and processing electric impulses.

This electrical activity can be obtained by the devices, which can measure EEG activity. The most popular companies interesting in the EEG are the Emotiv system and Neurosky. The devices from the Emotiv system was used in this research.

The selected device for obtaining electrical activity with the 14-channel scanner of EEG signals is called Emotiv EPOC+. It is a scalable system applied in context research of the human brain. Using this system can record, identify, interpret the brain activity and due to our control unit we can provide the ability to control external or inbuilt system (neuroprosthesis, rehabilitation aids, wheelchairs, household equipment, etc.). Our solution to a simple dynamic BCI system developed at the Faculty of Applied Informatics in Zlin, which are well applicable and universal. As knowledge of small desk computers Raspberry Pi and Arduino is utilized, compatibility with any sensors and communication environment is solved via $\mathrm{Wi}-\mathrm{Fi}$ and Bluetooth, following the current technological and transmission standards, approaching communication in real time. The accuracy of classification depends on the equipment sensitivity and adjustment to a concrete person. [4-7]

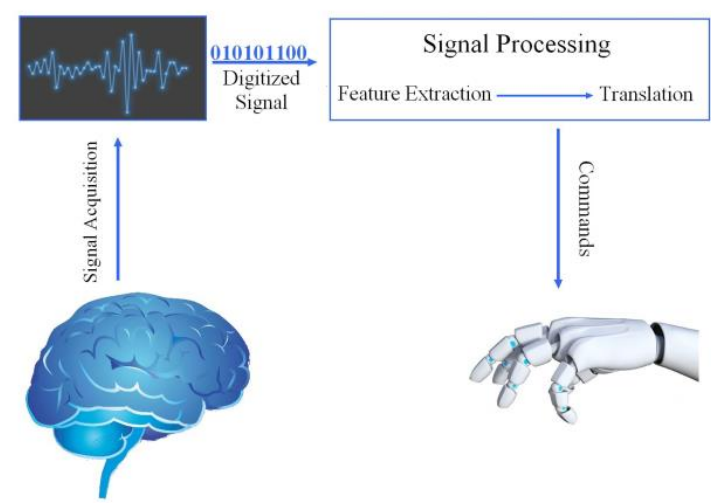

Fig. 1 Diagram of the BCI system (digitized signal of brain activity is processed and transformed into commands for the periphery devices included intelligent household, electric wheelchair, neuroprosthesis, equipment used for neurorehabilitation)

\footnotetext{
* Corresponding author: koudelkova@utb.cz
} 


\section{Monitoring of brain activity}

BCI technology has three types of implementation invasive, partially invasive and non-invasive. The implementations are based on scanning brain activity.

\subsection{Invasive BCI}

Invasive implantation of a BCI system means implanting of electrodes direct to grey matter. Contact of the electrodes with the tissue is direct. Therefore, the signal is the highest quality. However, the implementation in the brain requires an operation, which is not suitable for our research. Also applied to the invasive BCI can cause irreversible brain damage. Risks of the surgery are connected with craniotomy and postoperative changes accompanied by scar tissue formation. These time changes may weaken the signal or destroy it. [8]

\subsection{Partially invasive $\mathrm{BCl}$}

Partially invasive BCI system is also implemented intracranially. However, a part of the system rest outside of the brain, not in the grey matter. Partially invasive BCI system produces a worse quality of the signal than the invasive system. Nevertheless, the risk of brain tissue damage is lower. The typical representative of this technology is electrocorticography (further referred to as ECoG). [8]

\subsection{Non-invasive $\mathrm{BCl}$}

Non-invasive BCI system monitors brain signals through electrodes placed on the surface of the head. This system is called an electroencephalograph (EEG). The EEG is a record of temporal change in electrical potential, caused by brain activity. This record contains s broad frequency spectrum. The frequency range is usually specified between 0.1 and $100 \mathrm{~Hz}$. The suitable frequency for the BCI system was detected, which is the range up to $40 \mathrm{~Hz}$.

The non-invasive system is easy to use, but their major disadvantage is producing a poor quality of the signal because the skull dampens and disperses electromagnetic waves formed by the neurons. The problem mentioned above is solved by mathematical approximation algorithms implemented in research and scientific systems and kits included Emotiv EPOC+, which is used in this research. The first successful noninvasive BCI system was built in 1998. Doctor Christopher Guger built a system which, „taught“ the computer how to control 80 movements of both the left and the right hand using EEG. This system made use of 27 electrodes located on the surface of the head. [8-9]

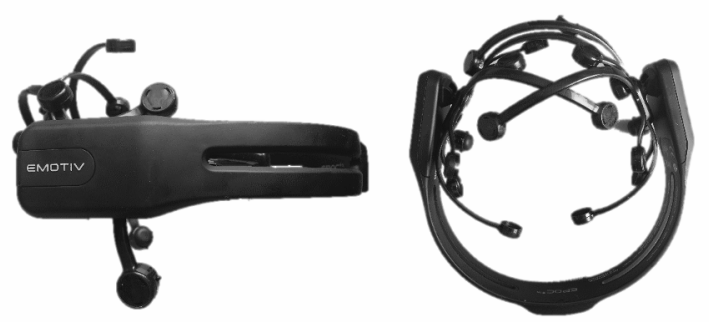

Fig. 2 EEG signals scanner Emotiv EPOC+

\section{Application of $\mathrm{BCl}$}

Currently, many research teams are working on function BCI applications. The focus is at least partial recovery of communication of the impaired human organism with the surroundings. Today, this functioning system using BCI which includes spelling systems, virtual limb or neuroprosthesis devices.

\subsection{Spelling systems}

These systems are designed for patients who are not able to communicate with their surroundings because of a combined phatic disorder. The most advanced systems make using ,steady state visually evoked potential“ (SSVEP). The EEG sensor of brain signals is interconnected with a computer. There were developed a unique application. The person chooses the group of symbols using SSVEP. The process is repeated until is choose a specific letter or symbol. This technology replaces simple verbal communication. [10-11]

\subsection{Virtual limb}

One of the BCI system applications which are used to compensate the ability to move with a limb is called the virtual hand. This application shows palms in the graphic interface. A patient is interconnected with the EEG signal sensor. The patient imagines the left or the right-hand movement. The sensor will evaluate electric signals coming from the brain, and the virtual palm moves the same as in the patient's mind. [10]

\subsection{Neuroprosthesis devices}

By processing and interconnecting the EEG signal with the neuroprosthesis, e.g., spinal cord lesions can be over bridged with the use of electric impulses. The first successful experiment using non-invasive BCI system to recover a palm function in a quadriplegic patient occurred in the year 2000. [10]

The mention examples indicate that not only the control actions for hardware platforms may be mapped universally, but also commands of the operating system or the system of keyboard shortcuts, without the presence of any further technological environment. The future solution to this research will be the introduction of a complex BCI in the field of applied robotics (e.g., neuroprosthesis in CNS injuries), remote controlled 
models operation (e.g., assistance robots), external devices or software operation (e.g., spelling system after CMP).

A very interesting application can also be a solution to the lock/unlocked in syndrome problem. These assumptions are also confirmed by the structure of the applications, which have been created at the Faculty of Applied Informatics, TBU in Zlin in recent years. New research direction focuses on hybrid systems. Interconnection of the BCI and EMG, ideas can be transformed into actions."

Combination of physiological EEG and EMG signals ranks among strong areas of interest in research on physiotherapy. The first system makes records of the brain activity, and the second system measures the activity inside the patient's muscles. The differences in EEG and EMG activities, compared to data in a healthy patient, may indicate the cause of loss motor skills function. This can be helpful, especially in different degrees of movement ability disorders, irrespective of the type of a handicap. In the future, we will aim to transform the findings inside our multi-branch team into different clinical applications in physiotherapy. [3, 12-15]

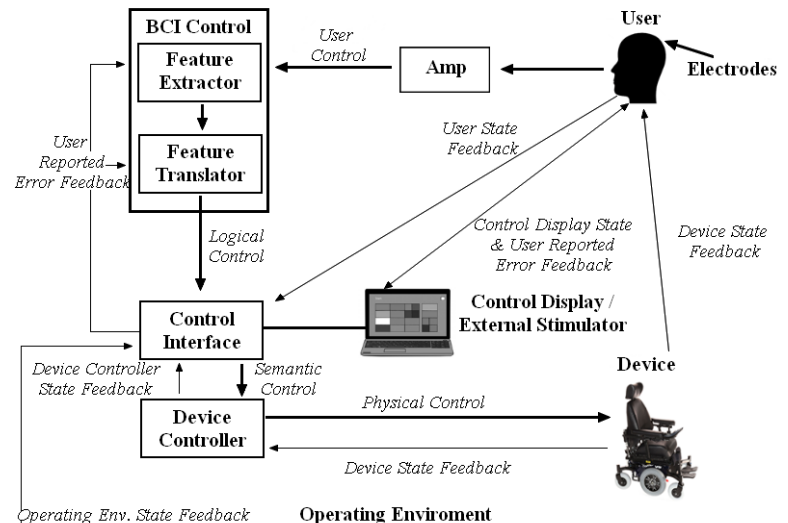

Fig. 3 Diagram for controlling external equipment by means of BCI

\section{Experimental part}

The aim of this section focuses on our research and methodology in the sphere of BCI.

The Faculty of Applied Informatics, Tomas Bata University in Zlin has been solving projects on BCI systems since 2008. Between 2016-2017 a team at the Faculty of Applied Informatics, TBU in Zlin developed a model of a non-invasive, simple BCI system serving to operate technical equipment as well as a robot built-up from intelligent components of a robotic kit with LEGO EV3. See in Figure 4.

External systems are controlled by activating mimic facial muscles, via the EEG sensor. It can be seen in Figure 2. The brain headset equipment by Emotiv system under the specification EPOC+, is a fourteen-channel EEG signal sensor, with two reference sensors - AF3, F7, F3, FC5, T7, P7, O1, O2, P8, T8, FC6, F4, F8, AF4. The device records signal in sequences, with a speed of 128
$\mathrm{Hz}$, with sufficient resolution of 14 bites in one channel and frequency response within the range of 0.16-43 $\mathrm{Hz}$. Besides, it enables wireless transfer of data, with a frequency of $2.4 \mathrm{GHz}$, with a battery enabling continuous work for eight hours. One of the assets of the equipment is its possibility to use a saline solution instead of a conventional conductive gel, to build contact between the electrodes and the head skin. [20]

The Emotiv Epoc+ is a scientific and research device which guarantees sufficient, and not only essential accuracy with its parameters (for the list of specific parameters of the current system see the reference [20]). Our team aims to verify the abilities of the sensor and apply them in clinical research, which is currently being done.

When the technical design was being developed, the risk of a low-quality signal, described in the literature on non-invasive applications, was removed successfully. The system was tested. Dissertation, theses, and articles were published. See the reference list. From this information was built a multi-branch team. This team included technicians, doctors, and physiotherapists with a focus on the future application of BCI technologies in clinical practice. This team can help patients with permanent impairment of the nervous system, such post-traumatic states, degenerative and metabolic disorders, strokes and other neurological diseases. During the first stage, the team focused on the ability to catch (measure) digitallyprocessed signals using BCI.

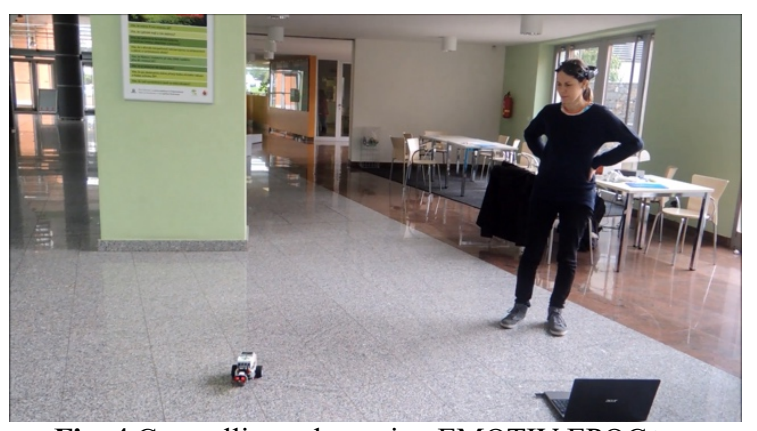

Fig. 4 Controlling robot using EMOTIV EPOC +

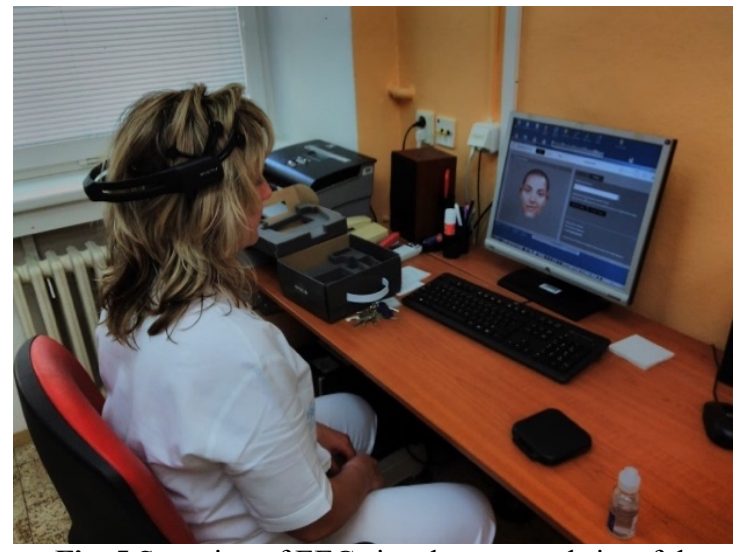

Fig. 5 Scanning of EEG signals on a worksite of the Clinic of Rehabilitation and Physical Medicine, University Hospital in Ostrava 
At the current stage of the research, we can register and subsequently evaluate the measurement outputs, which are based on multidisciplinary cooperation. The signals are processed and evaluated within a more extended period (months). After that, the team will deal with the possibilities of the knowledge application in practice. It means there is the area of objective evaluation of physiotherapy, utilizing recognizable activities of brain regions or evaluating the feedback process in patients with various types of impairment of the nervous system during the complex physiotherapeutic treatments. It belongs to exercise on machines, ergotherapy, psychology, and other methods. This information has been confirmed by the results of international research studies focusing on these problems. See the reference list [16-19].

\section{Conclusion}

The technology of the BCI system is a relatively new research area, with broad application potential. This system can be helpful in the possibility of improving the quality of life in patients with permanent neurological disease. Up to now much research focused on this neuroinformatics area can be traced. However, in the field of clinical physiotherapy research activities are at the beginning, and their possibilities are unknown. The patient suffering from a neurological deficit has a possibility of not only a passive rehabilitation, but also of active help of ,concentrated attention - the strength of ideas". The first findings in our multi-branch cooperation with still relatively simple BCI applications indicate new possibilities in this sphere of medicine.

This work was supported by Internal Grant Agency of Tomas Bata University in Zlin under the project No. IGA/FAI/2019/005.

\section{References}

[1] J. J. Shih, D. J. Krusienski, J. R. Wolpaw, Mayo Clinic Proceedings, 87, 3, pp. 268-279, (2012).

[2] S. Amiri, R. Fazel-Rezai, V. Asadpour, Advances in Human-Computer Interaction, 2013, 1-8, (2013).

[3] EEG and EMG in rehabilitation. Ant neuro Available: https://www.ant-neuro.com/showcase/eeg-and-emg-rehabilitation (2019).

[4] J. Svejda, Dissertation thesis, TBU in Zlin, 84 pages, ISBN 978-80-7454-683-9, (2013).

[5] P.R. Kennedy, et al., IEEE Transactions on Rehabilitation Engineering, 8 (2), 198-202, (2000).

[6] W. H. Pilcher, D. L. Silbergeld, M. S. Berger, and G. A. Ojemann, Journal of Neurosurgery, 78, 891-902, (1993).

[7] R. N. Khushaba, C. Wise, S. Kodagoda, J. Louviere, B. E. Kahn, and C. Townsend, Expert Systems with Applications, 40, 3803-3812, (2013).
[8] H. Anupama, G.M. Limgaraju, International Journal of Advances in Engineering \& Technology, 3, 739745, (2012).

[9] H. Cecotti, Journal of Physiology-Paris, 105, pp. 106-114, (2011).

[10] G. Pfurtscheller, G. R. Muller-Putz, R. Scherer, and C. Neuper, Rehabilitation with Brain-Computer Interface Systems, Computer, 41, 58-65, (2008).

[11] X. Chen, Y. Wang, M. Nakanishi, X. Gao, T.-P. Jung, and S. Gao, Proceedings of the National Academy of Sciences, 112, pp. E6058-E6067, (2015).

[12] R. Zak, Dissertation thesis, TBU in Zlin, 61 p., ISBN 978-80-7454-685-3, (2013).

[13] Biomechatronics in medical rehabilitation. New York, NY: Springer Berlin Heidelberg, ISBN 9783319528830, (2017).

[14] J. W. L. Pau, S. S. Q. Xie, A. J. Pullan, IEEE Transactions on Biomedical Engineering, 59, 9, pp. 2586-2593, (2012).

[15] K. Knaepen, P. Beyl, S. Duerinck, F. Hagman, D. Lefeber, R. Meeusen, IEEE Transactions on Neural Systems and Rehabilitation Engineering, 22, 6, pp. 1128-1137, (2014).

[16] R. N. Khushaba, L. Greenacre, S. Kodagoda, J. Louviere, S. Burke, and G. Dissanayake, Expert Systems with Applications, 39, 16, pp. 12378-12388, (2012).

[17] G. S. Taylor and C. Schmidt, Proceedings of the Human Factors and Ergonomics Society Annual Meeting, 56, 1, pp. 193-197, (2012) .

[18] P. Bobrov, A. Frolov, C. Cantor, I. Fedulova, M. Bakhnyan, and A. Zhavoronkov, PLoS ONE, 6, no. 6, p. e20674, (2011).

[19] E. W. Anderson, K. C. Potter, L. E. Matzen, J. F. Shepherd, G. A. Preston, and C. T. Silva, Computer Graphics Forum, 30, 3, pp. 791-800, (2011).

[20] Emotiv: Emotiv Epoc+, Available on: https://www.emotiv.com/product/emotiv-epoc14-channel-mobile-eeg/ 